OPEN ACCESS

Edited by:

Jessica L. Jones,

United States Food and Drug

Administration, United States

Reviewed by:

Aye Moa,

University of New South

Wales, Australia

Carmelo Massimo Maida,

University of Palermo, Italy

*Correspondence:

Rong-Bao Gao

rongbaogao@163.com

Hai-Feng Pan

panhaifeng@ahmu.edu.cn;

panhaifeng1982@sina.com

tThese authors have contributed equally to this work and share first authorship

Specialty section:

This article was submitted to Infectious Diseases - Surveillance,

Prevention and Treatment,

a section of the journal

Frontiers in Public Health

Received: 30 November 2021

Accepted: 19 January 2022

Published: 24 February 2022

Citation

He J, Hou S, Chen Y, Yu J-L, Chen Q-Q, He L, Liu J, Gong L,

Huang $X-E, W U$ J-B, Pan H-F and Gao R-B (2022) The Epidemiological Pattern and Co-infection of Influenza $A$ and $B$ by Surveillance Network From 2009 to 2014 in Anhui Province, China.

Front. Public Health 10:825645. doi: 10.3389/fpubh.2022.825645

\section{The Epidemiological Pattern and Co-infection of Influenza A and B by Surveillance Network From 2009 to 2014 in Anhui Province, China}

\author{
Jun He ${ }^{1,2 t}$, Sai Hou ${ }^{1,2 \dagger}$, Yue Chen ${ }^{3,4 t}$, Jun-Ling Yu ${ }^{1,2}$, Qing-Qing Chen ${ }^{1,2}$, Lan He ${ }^{1,2}$, \\ Jiang Liu ${ }^{5}$, Lei Gong ${ }^{1,2}$, Xin-Er Huang ${ }^{6}$, Jia-Bing Wu ${ }^{1,2}$, Hai-Feng Pan ${ }^{3,4 *}$ and \\ Rong-Bao $\mathrm{Gao}^{7 *}$
}

${ }^{1}$ Microbiological Laboratory, Anhui Provincial Center for Disease Control and Prevention, Hefei, China, ${ }^{2}$ Microbiological Laboratory, Public Health Research Institute of Anhui Province, Hefei, China, ${ }^{3}$ Department of Epidemiology and Biostatistics, School of Public Health, Anhui Medical University, Hefei, China, ${ }^{4}$ Inflammation and Immune Mediated Diseases Laboratory of Anhui Province, Hefei, China, ${ }^{5}$ Huainan City Center for Disease Control and Prevention, Huainan, China, ${ }^{6}$ Department of Health Inspection and Quarantine, School of Public Health, Anhui Medical University, Hefei, China, ${ }^{7}$ NHC Key Laboratory of Biosafety, NHC Key Laboratory of Medical Virology and Viral Diseases, National Institute for Viral Disease Control and Prevention, Chinese Center for Disease Control and Prevention, Beijing, China

Influenza-like illness (ILI) is one of the most important public health problems globally, causing an enormous disease burden. Influenza infections are the most common cause of ILI. Bacterial and virus co-infection is common yet the data of co-infection with influenza $A$ and $B$ viruses are scarce. To identify the epidemiological patterns of and co-infection of influenza $A$ and $B$ in Anhui province, China, we analyzed the surveillance data of 5 years from 2009 to 2014 collected by the Chinese National influenzas network. The results showed that the weekly ratio of ILI was $3.96 \pm 1.9 \%$ (95\% Cl 3.73-4.2\%) in outpatients and the highest affected population was children under 5 years old. The epidemic of influenza viruses was highest during 2009-2010. For the other 4 surveillance years, school-aged people (5-14 years) were the most highly affected population. Influenza B and H3N2 viruses were more prevalent than H1N1pdm09 virus after 2010. In addition, a significant co-circulation of influenza A (H1N1pdm09 and H3N2) and influenza B virus was detected with $0.057 \%$ PCR positive rate during 2009-2014 in Eastern China, yet isolated only in pediatric patients. Our data reveals school-aged population would be the main vulnerable population and a distinct seasonality for influenza. In addition, the co-infection of influenza A and B were found in Anhui Province, China. Ongoing surveillance is critical to understand the seasonality variation and make evidence-based vaccination recommendations. Information on the epidemiological patterns and co-infections of influenza A and B can help us to implement different strategies for selecting vaccine formulations and monitoring new emerging influenza strains. In addition, the identification of the susceptible population can help us to develop more precise protection measures.

\footnotetext{
Keywords: influenza-like illness, H1N1pdm09, H3N2, influenza B, co-infection
} 


\section{INTRODUCTION}

Influenza virus, one of the most important respiratory viruses presenting globally annual seasonal epidemics, causes fatal respiratory diseases to humans. The estimated number of global annual influenza-associated respiratory deaths were 291,243645,832 per year during the 1999-2015 period, and 9,243105,690 deaths occur annually among children younger than 5 years (1). The mortality related to influenza virus infections adhere to a cyclic pattern fluctuating with seasonal changes in temperature and humidity, or occasional pathogenicity changes due to new gene mutations or exchanges (2-5). It is wellrecognized that bacterial co-infection was frequent and critically led to the mortality in influenza virus infection $(6,7)$. In contrast, previous reports indicated that medical manifestations in cases of dual influenza infection and simple infection are similar (8). Recent ferret model study implied that prior infection antigenically related and unrelated viruses protected from subsequent infection or modified the infection kinetics of the challenge virus (9). The epidemiological and virological study of dual influenza infection cases is of major interest particularly for monitoring emerging influenza strains, which could perpetuate epidemic or pandemic events. However, coinfection with different influenza type/subtype viruses is rare $(8,10)$, and remain to be fully elucidated due to some limitations of many studies. Co-infections with influenza A subtypes or/and influenza B virus were detected in several surveillance areas regarded as rare events (11). The epidemic of influenza in Anhui Province, China generally exhibited Influenza $\mathrm{B}, \mathrm{H} 3 \mathrm{~N} 2$ and H1N1pdm09 alternating or co-prevalence trend and presented obvious seasonal characteristics, showing single and double peaks, i.e., the peak is bound to occur every winter and spring, while the summer peak occurs in alternate years. In the present study, we described the epidemiological pattern of influenza A and B by surveillance network in Anhui province, eastern China from 2009 to 2014. In addition, we also investigated co-infection of influenza A and B and identified the ssusceptible population.

\section{MATERIALS AND METHODS}

\section{Source of Data}

Influenza surveillance has been established and developed a surveillance network in Anhui Province of Eastern China since 1990. The network has covered 17 collaborating laboratories of local CDCs and 24 sentinel hospitals until 2009. Based on the guideline of Chinese influenza surveillance (12), the annual surveillance begins at the 14th week (April) of present year and ends at the 13th week (March) of next year, and the collaborating laboratories collected samples from patients with ILI for virological surveillance in sentinel hospitals weekly. Referring physicians at sentinel hospitals were asked to diagnose ILIs according to strict criteria (fever $>38^{\circ} \mathrm{C}$, cough or sore throat) and to record the number of

Abbreviations: ILI, influenza-like illness; HI, haemagglutination inhibition assay; HA, hemagglutinin.
ILI consultations per day in a fixed format based on age group. These data would be uploaded into Chinese influenza surveillance informatics system by designated hospital staff in hospitals daily. Pharyngeal swab specimens were collected from patients without taking antiviral drugs within 3 days of illness onset. The specimens were transported to the correspondent laboratories in viral transport medium at $4{ }^{\circ} \mathrm{C}$ for RT-PCR detection and/or viral isolation according to Chinese influenza surveillance guidelines (12). The detection results including weekly type and subtype specific positive rates were input into Chinese influenza surveillance informatics system by collaborating laboratories. In the present study, we collected pharyngeal swab specimens and surveillance data of 5 years from 2009 to 2014 from 17 collaborating laboratories of local CDCs and 24 sentinel hospitals in Anhui Province of China.

\section{RNA Extraction and RT-PCR}

Virus RNA were extracted by RNeasy Mini kit (Qiagen) in pharyngeal swab specimens collected from sentinel hospitals, according to the manufacturers' recommendations. Then we took specific real-time RT-PCR assays for seasonal influenza viruses (H1N1pdm09, H3N2 and influenza

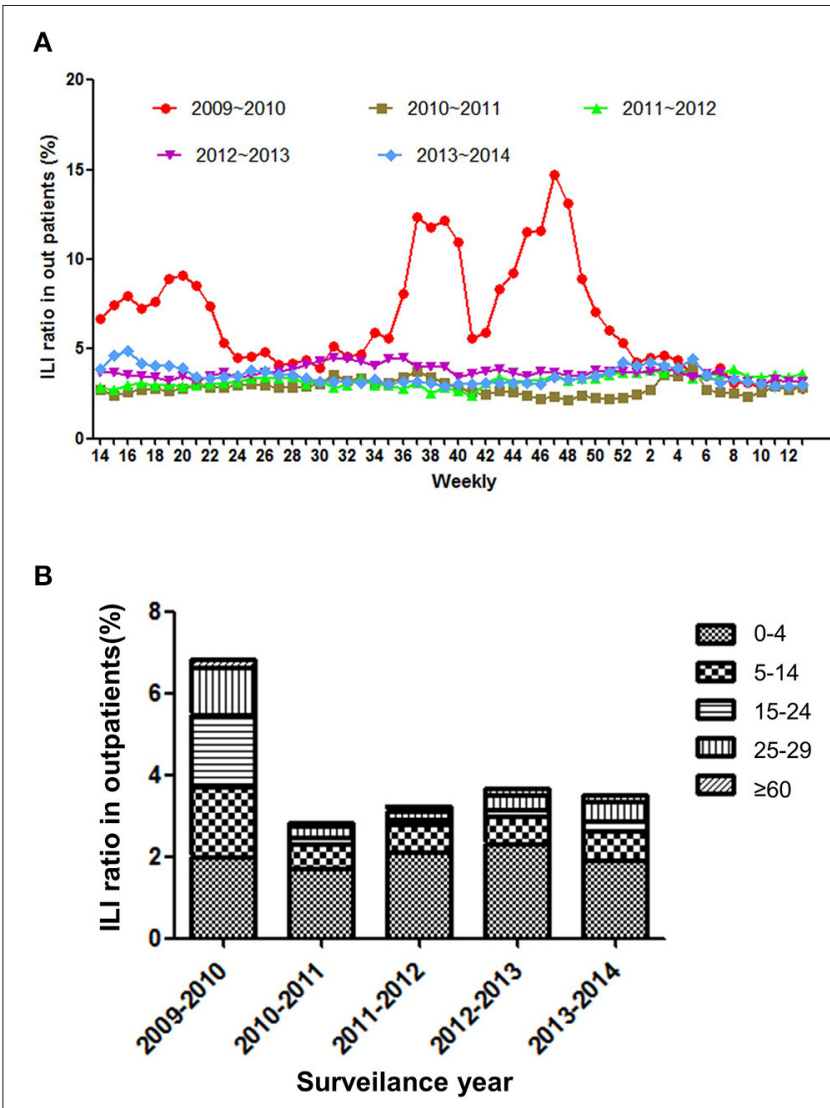

FIGURE 1 | (A) The weekly ratio of ILI outpatients from 2009-2014. (B) The incidence of ILI outpatients by age group from 2009-2014. 
B). The nucleic acids of the virus were extracted by GeneRotex Automatic Nucleic acid extractor (TIANLONG Technology CO. LTD). The identification of influenza virus was carried out by QuantStudio Q5 System (Applied Biosystems, Waltham, MA, USA) using AgPath-ID Onestep RT-PCR kit (Applied Biosystems, Foster City, CA), the primers and the fluorogenic probes were synthesized according to the national influenza surveillance program (2017 version).

\section{Isolation of the Virus and Identification of Type/Subtype}

The samples of pharyngeal swab were maintained in a viral-transport medium and propagated in MDCK cells for $72 \mathrm{~h}$ at $35^{\circ} \mathrm{C}$. The supernatant was tested by using hemagglutinin (HA) assay with human "O" type red blood cells, and influenza type and subtype was performed for those with positive hemagglutinin inhibition tests or PCR. The extracted RNA was first tested for the presence of influenza A and B viruses. The
A

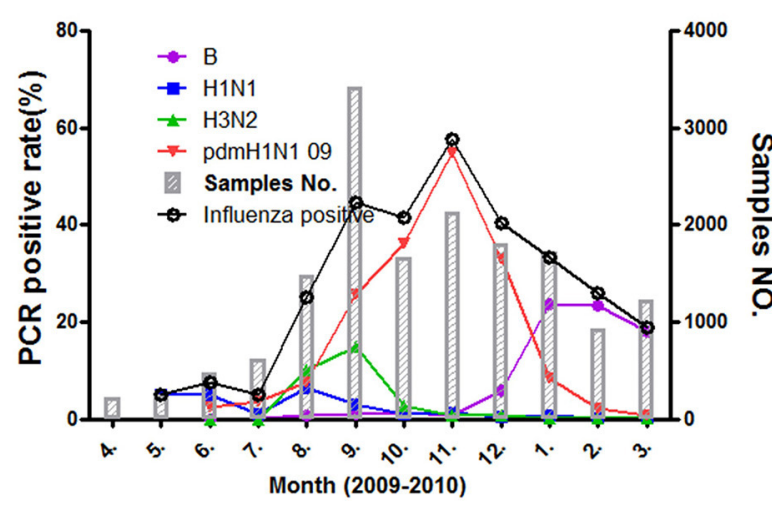

C

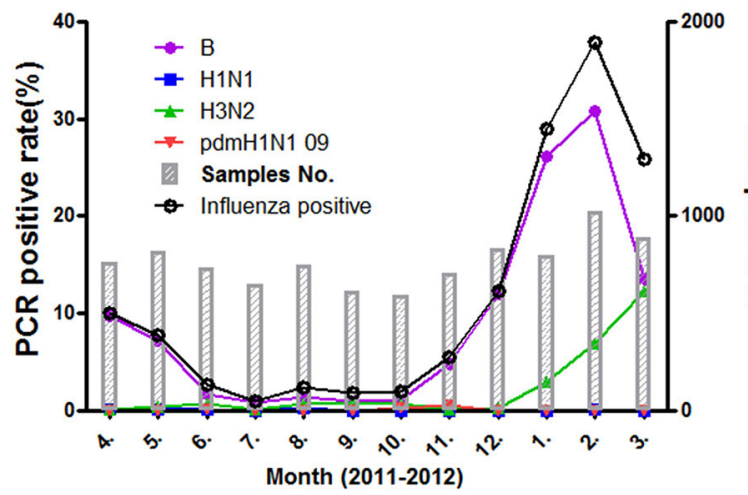

E

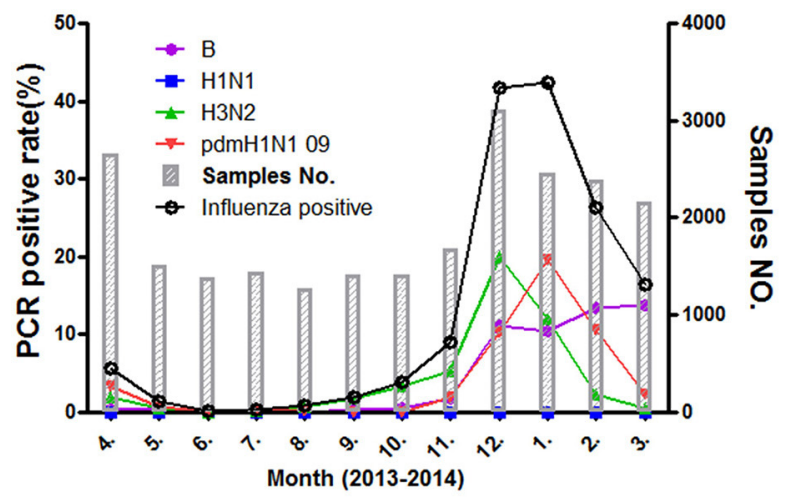

B

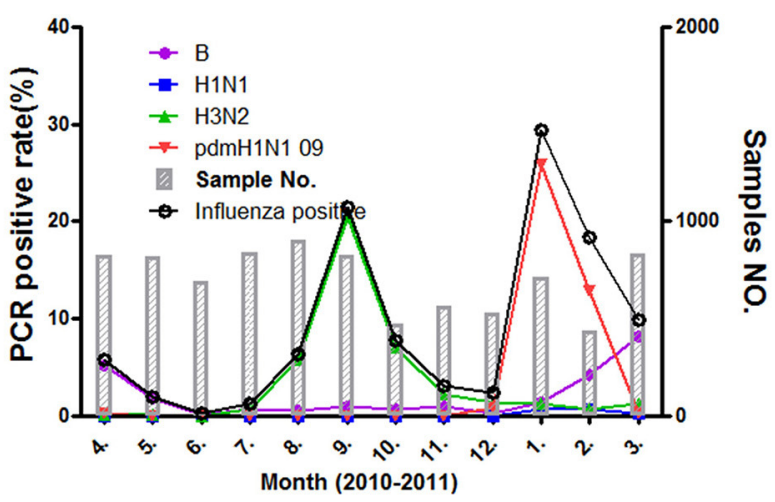

D

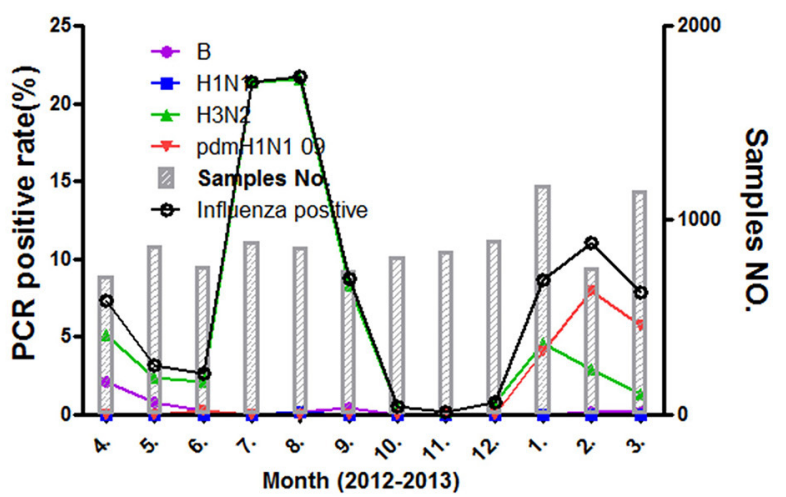

FIGURE 2 | The positive rate of different influenza subtypes during the different surveillance years (A-E) from 2009 to 2014. 
nucleic acid showing positive for influenza A/B was further differentiated into subtypes and lineages. Influenza A positive samples were screened for $\mathrm{H} 1 \mathrm{pdm}$ and $\mathrm{H} 3$ whereas samples showing influenza B positive were tested for B/Yamagata and $\mathrm{B} /$ Victoria lineage.

\section{Haemagglutination Inhibition Assay (HI)}

HI was performed as previously described (13). Reference sera were handled with a 1:4 ( $\mathrm{vol} / \mathrm{vol})$ of receptor disrupting enzyme for $18 \mathrm{~h}$ at $37^{\circ} \mathrm{C}$ and then incubated at $56^{\circ} \mathrm{C}$ for $30 \mathrm{~min}$. The reference serum was titrated with a 2 -fold dilution of PBS (initial dilution of 1:20) and then $4 \mathrm{HAU} / 25 \mu \mathrm{l}$ virus was added for the assay.

\section{Data Analyses}

The chi-squared with Fisher's exact test was used to compare dichotomous variables between groups using Graph
Prism 5 software (GraphPad Software), and $P<0.05$ was considered significant.

\section{RESULTS}

\section{Consultation of ILI in Anhui Province}

The total number of consultations of ILI was 5,62,551, and the age group percentages by year of surveillance are presented in Supplementary Table 1 . The results showed that weekly ILI ratio of outpatients was $3.96 \pm 1.9 \%$ (95\% CI: $3.73-4.2 \%$ ) for the 5 surveillance years and were the highest in surveillance year of 2009-2010 (6.75 $\pm 3.05 \%$, 95\%CI: 5.72-7.43\%). In addition, there were three peaks at 20th, 38th, and 50th weeks during 20092010 but not presented in subsequent same seasons (Figure 1A). As is shown in Figure 1B, the youngest patients $(<5$ years old) presented the highest ILI consultations $(2.01 \pm 0.22 \%$, 95\% CI: $1.74-2.28 \%$ ). In contrast, the $>65$ years old population

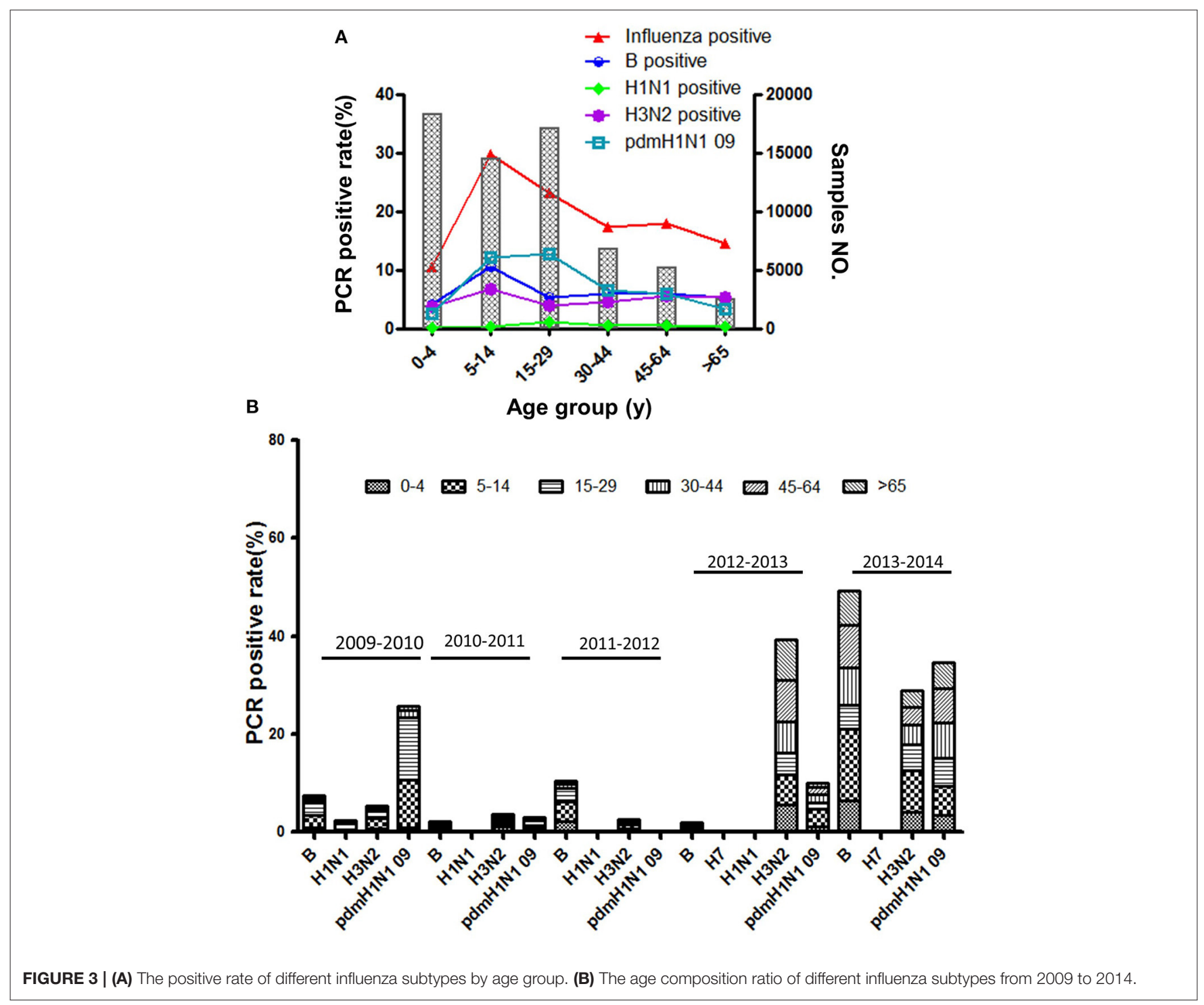


was minimum ILI consultation $(0.15 \pm 0.05 \%$, 95\% CI: $0.08-$ $0.21 \%$ ) during the influenza epidemic period. To be different in other surveillance years, the ILI consultation of in 2009-2010 present significantly higher level in senior school-aged (15-29 years) population than in school-aged (5-15 years) children $(P$ $<0.0001$, OR $=0.95$ ).

\section{Epidemic of Influenza Viruses}

The epidemic of influenza viruses showed fluctuation in different surveillance year, and the cycling pattern present big difference in these surveillance years. As is shown in Figure 2, the activities of influenza viruses were the strongest in 2009-2010 and kept a high level for long period (July 2009-March 2010) and peaked in November. In addition, three peaks (located in spring, summer, and winter respectively) were present in 2010-2011 and 20122013. Both summer peaks were raised by H3N2 influenza virus, whereas only spring and winter peaks presented in 20112012 and 2013-2014. In those spring and winter epidemic peaks, influenza A (H1N1pdm09, H3N2) and B exhibited cocirculation. Of note, the novel H7N9 avian influenza virus infection was spotted among ILI patients in 2013 and 2014 by the surveillance system.

\section{Relative Rate of Influenza Infection}

Based on standard clinical groupings according to potential influenza exposure/risk environments, the 5 years surveillance data showed the influenza consultation difference in different age ILI patients. As is shown in Figure 3A, the influenza infections were the highest in young school-aged patients $(24.25 \pm 16.52 \%$, 95\% CI: $3.74-44.77 \%)$, then in senior school-aged people (18.34 $\pm 16.14 \%$, 95\% CI: $-1.70-38.38 \%)$. To be different with ILI consultation, influenza infection was significantly lowest in the youngest ( $<5$ years old) patients $(10.78 \pm 5.77 \%$, 95\% CI: $3.61-$ $17.95 \%)$, and then in oldest ( $>65$ years old) patients $(14.36 \pm$ 5.219\%, 95\% CI: 7.88-20.84\%). However, the consultation of senior school-aged patients was significantly decreased (11.29 $\pm 4.01 \%$, 95\% CI: $4.91-17.68 \%$ ) if we took out pandemic year (2009-2010) data (Supplementary Figure 7). In addition, the H1N1pdm09 virus was the most prevalent influenza virus in ILI patients (Figure 3B), especially in school-aged people (5-29 years old). However, Influenza B and $\mathrm{H} 3 \mathrm{~N} 2$ virus were more prevalent than H1N1pdm09 if pandemic year (2009-2010) surveillance data was taken out $(P<0.001, O R=1.51)$. More details information associated with total ILI consultations by age group and surveillance year from 2009 to 2014 years were presented in Supplementary Figures 1-7; Supplementary Tables 1, 2.

\section{Co-infection With Influenza A and B in ILI Patients}

The co-infection of influenza viruses was detected in ILI patients by PCR during the five surveillance years. The detected rate of co-infection was $0.057 \%$ in all collected ILI samples, and $0.58 \%$ in influenza PCR positive samples. To be consistent with influenza epidemic season, the positive co-infection peaked in the winter, summer or spring (Figure 4).

To define viral features of co-infection, the available samples were collected for viral isolation. Nine live viruses were isolated

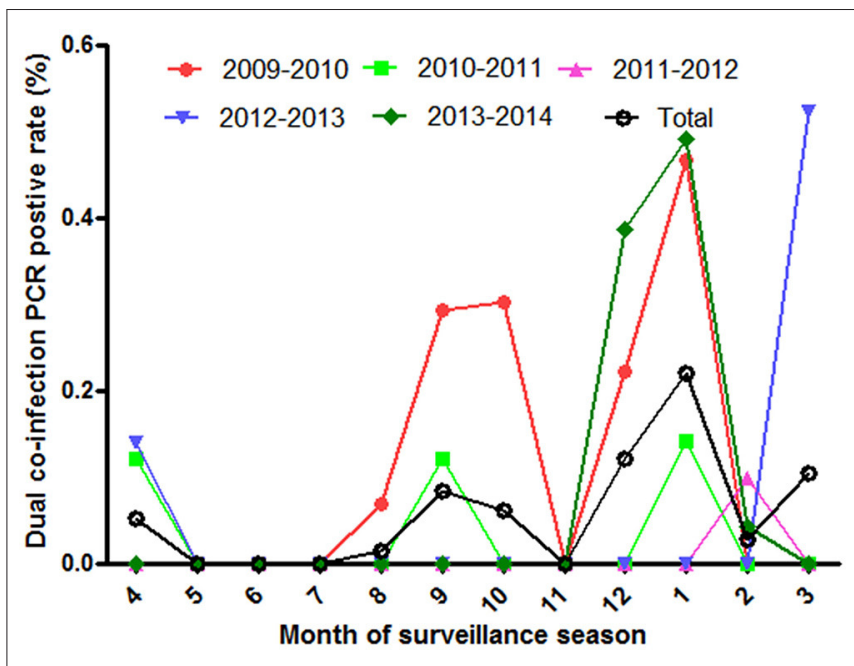

FIGURE 4 | The positive rate of influenza co-infection in different surveillance months from 2009 to 2014.

from 25 PCR-positive samples, four of which had detectable HI titers for dual viruses, including two H1N1pdm09 combined with Influenza B (Yamagata) and two H1N1pdm09 combined with $\mathrm{H} 3 \mathrm{~N} 2$ infection, respectively (Table 1). All of these 4 samples were collected from pediatric patients. The remaining five samples were isolated with only a single virus with detectable HI titers.

\section{DISCUSSION}

Effective influenza surveillance systems could help us decipher epidemiology and seasonality of influenza and further optimize possible influenza control strategies. The ILI consultation rates reported by Chinese influenza surveillance network in Anhui province of eastern China showed that ILI affected all aged people through whole year and was remarkably higher in 2009-2010 than in other surveillance years. To be consistent with ILI epidemics, influenza positive rates were the highest in 2009-2010 since H1N1pdm09 outbreak $(14,15)$. In addition, the data showed that ILI consultation were the highest in children aged $<$ 5 years. However, lab detection showed that influenza infections were the most predominant in school aged patients in the surveillance years instead of the youngest patients. In contrast, the most slightly affected people were the youngest patients among the monitored population. The data suggested that other respiratory pathogens may induce youngest ILI patients more frequently than influenza viruses as recent report $(16,17)$, and school-aged children should be the mainly concerned points for influenza controls in Anhui province China. In addition, the senior school-aged people were more increasingly affected during pandemic year, that is consisted with the previous reports where inpatients in the pandemic season were significantly younger compared with those in the pre-pandemic or postpandemic seasons (18-20). It may be partially associated with 
TABLE 1 | Co-infection with influenza A and B in ILI patients enrolled.

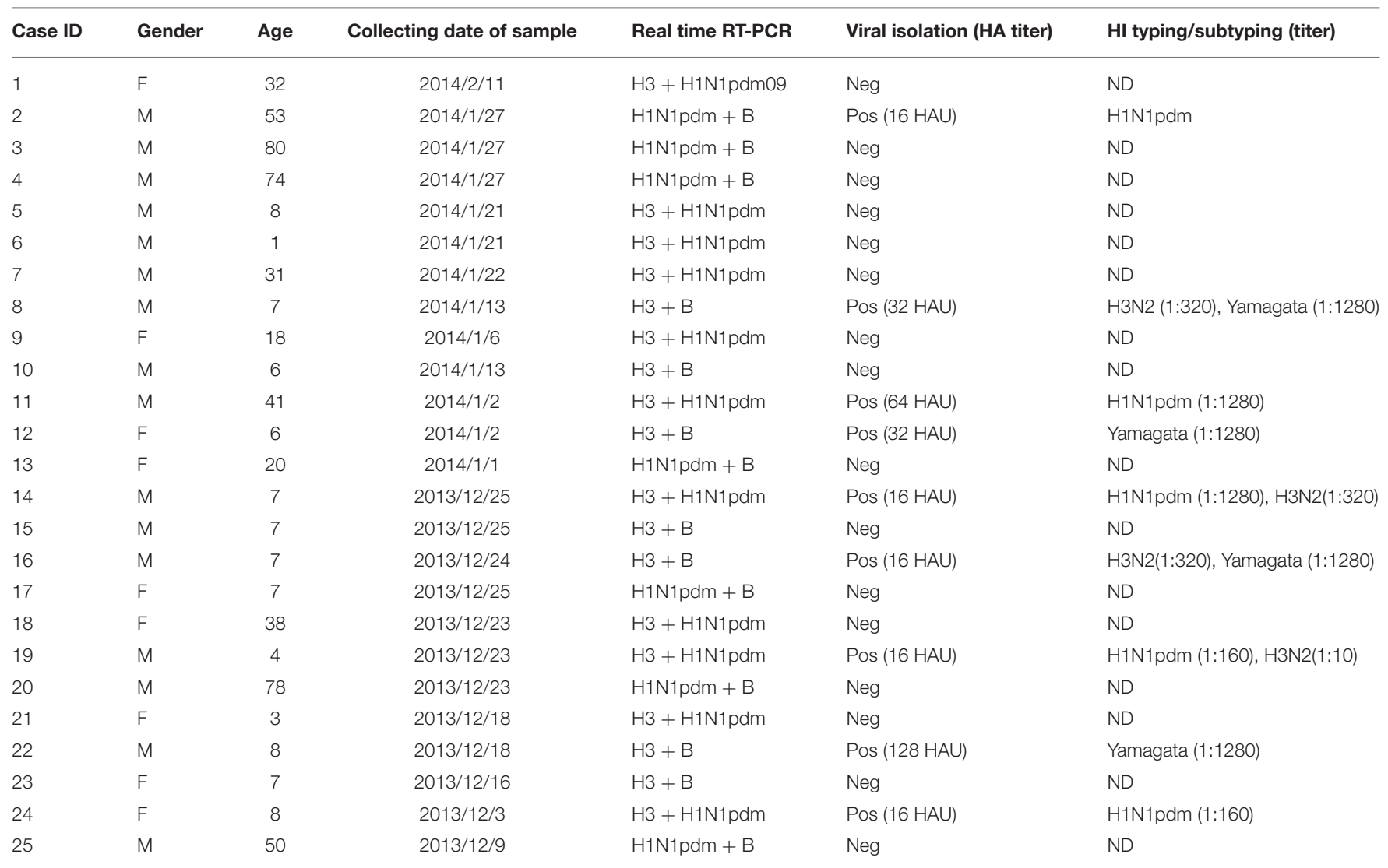

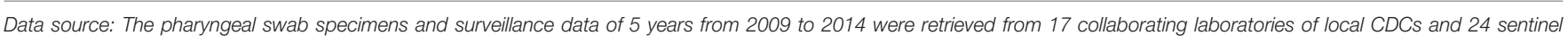
hospitals in Anhui Province of China.

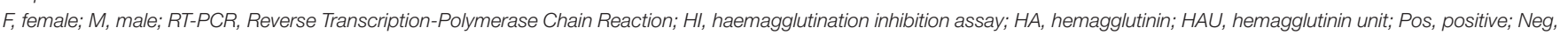
negative; $N D$, not detected.

that young adult in the pandemic season were less likely to have been vaccinated (20).

Influenza seasonality in Anhui province China was manifested by seasonal variations in different surveillance years. Except 2009-2010 surveillance year with H1N1 pandemic, two different epidemic patterns were present in the post the 4 surveillance years. Besides the normal winter and spring peaks, a summer peak presented in both 2010-2011 and 2012-2013 surveillance year. Similar recommendations of vaccination have been proposed for China recently (21). Recent data from countries in south and south-eastern Asia also indicate that countries consider vaccination with newly recommended vaccine in May-June prior to seasonal epidemic (22). In addition, the surveillance system presented a high sensitivity on monitoring the activity of the influenza viruses. An H7N9 case who was one of the earliest confirmed cases in mainland China was reported by the system $(23,24)$. Consequently, the surveillance networks would play a positive role in making evidence-based policy of control and prevention.

In the present study, human co-infections caused by several influenza viruses were detected in the surveillance epidemics.
However, live dual viruses were merely detected in a few cases. It means that there was a prepotent virus in the infections with dual influenza viruses associated with viral interference or cross-reactive immune responses (25). Infections with heterosubtypic influenza A viruses presented shortening of virus shedding in previous studies (26). In addition, Crossreactive epitopes have been found between influenza $A$ and $B$ viruses in the fusion peptide of the $\mathrm{HA}$ and enzymatic region of neuraminidase (25). Notably, the possibility of dual virus infection with similar replication level in vivo may be more frequent in young patients, that may be associated with host immune response to the infected virus. Previous studies shed a light on the immune response to influenza virus was weaker in the younger children and those without preexisting immunity (27). Theoretically, dual influenza virus infections represent a potential source of multiple viral transmission and constitutes a basis for virus recombination between two human strains or one human and one avian viral strain $(28,29)$. So, it may be valuable to monitor the potential reassortment due to co-infection in pediatric patients or those without preexisting immunity. 


\section{CONCLUSION}

In the aggregate, we elaborated two different patterns of influenza circulation in different surveillance years in Anhui province, China. In view of school-aged children were the mainly affected patients in ILI associated with influenza viruses, we propose to implement a vaccination program in school-aged population in Anhui province. Influenza B, H3N2 and H1N1pdm09 cocirculated in annual epidemics, and the co-infections with influenza viruses were frequent in epidemic peaks. These data help us implement strategies to select vaccine formulations and monitor potential recombinant viruses in surveillance programs.

\section{DATA AVAILABILITY STATEMENT}

Influenza surveillance data used and analyzed in the current study are available from the corresponding authors on reasonable request.

\section{AUTHOR CONTRIBUTIONS}

R-BG and H-FP conceptualized the review. Literature search was performed by J-BW and X-EH. Supervision was performed by

\section{REFERENCES}

1. Iuliano $\mathrm{AD}$, Roguski KM, Chang $\mathrm{HH}$, Muscatello DJ, Palekar R, Tempia S, et al. Estimates of global seasonal influenzaassociated respiratory mortality: a modelling study. Lancet. (2018) 391:1285-300. doi: 10.1016/S0140-6736(17)33293-2

2. Chadha MS, Potdar VA, Saha S, Koul PA, Broor S, Dar L, et al. Dynamics of influenza seasonality at sub-regional levels in India and implications for vaccination timing. PLoS ONE. (2015) 10:e0124122. doi: 10.1371/journal.pone.0124122

3. Linde A, Rotzen-Ostlund M, Zweygberg-Wirgart B, Rubinova S, Brytting M. Does viral interference affect spread of influenza? Euro Surveill. (2009) 14:19354. doi: 10.2807/ese.14.40.19354-en

4. Greenbaum BD, Li OT, Poon LL, Levine AJ, Rabadan R. Viral reassortment as an information exchange between viral segments. Proc Natl Acad Sci USA. (2012) 109:3341-6. doi: 10.1073/pnas.1113300109

5. Lam TT, Hon CC, Pybus OG, Kosakovsky Pond SL, Wong RT, Yip CW, et al. Evolutionary and transmission dynamics of reassortant H5N1 influenza virus in Indonesia. PLoS Pathog. (2008) 4:e1000130. doi: 10.1371/journal.ppat.1000130

6. Cauley LS, Vella AT. Why is coinfection with influenza virus and bacteria so difficult to control? Discov Med. (2015) 19:33-40.

7. Ackerman E, Longini IM Jr, Seaholm SK, Hedin AS. Simulation of mechanisms of viral interference in influenza. Int J Epidemiol. (1990) 19:44454. doi: 10.1093/ije/19.2.444

8. Almajhdi FN, Ali G. Report on influenza A and B viruses: their coinfection in a Saudi leukemia patient. BioMed Res Int. (2013) 2013:290609. doi: 10.1155/2013/290609

9. Laurie KL, Guarnaccia TA, Carolan LA, Yan AW, Aban M, Petrie S, et al. Interval between infections and viral hierarchies are determinants of viral interference following influenza virus infection in a ferret model. J Infect Dis. (2015) 212:1701-10. doi: 10.1093/infdis/jiv260

10. Falchi A, Arena C, Andreoletti L, Jacques J, Leveque N, Blanchon T, et al. Dual infections by influenza A/H3N2 and B viruses and by influenza A/H3N2 and A/H1N1 viruses during winter 2007, Corsica Island, France. J Clin Virol. (2008) 41:148-51. doi: 10.1016/j.jcv.2007.11.003
LG and JL. The first draft and editing of the manuscript were performed by JH, SH, and YC. Visualization/data presentation were performed by J-LY, Q-QC, and LH. All authors commented on previous versions of the manuscript, read, and approved the final manuscript.

\section{FUNDING}

This work was supported by the Scientific Research Projects of Health Commission of Anhui Province in 2021 (grant number: AHWJ2021a030).

\section{ACKNOWLEDGMENTS}

We appreciate the Chinese influenza surveillance network for providing the clinical samples and data collected from patients.

\section{SUPPLEMENTARY MATERIAL}

The Supplementary Material for this article can be found online at: https://www.frontiersin.org/articles/10.3389/fpubh. 2022.825645/full\#supplementary-material

11. World Health Organization. Influenza Update. (2015). Available online at: https://www.who.int/en/news-room/fact-sheets/detail/influenza-(seasonal). (accessed May 15, 2015).

12. National Health Commission of the People's Republic of China. Guideline of Chinese Influenza Surveillance. (2017). Available online at: http://www.nhc. gov.cn/wjw/index.shtml (accessed March 30, 2017).

13. Xu C, Bai T, Iuliano AD, Wang M, Yang L, Wen L, et al. The seroprevalence of pandemic influenza H1N1 (2009) virus in China. PLoS ONE. (2011) 6:e17919. doi: 10.1371/journal.pone.0017919

14. Wei M, Yan Z, Wang C, Liu W, Cao W. Eight-hospital based influenza like illness surveillance from April, 2009 to March, 2011 in China. Influenza Other Respir Viruses. (2013) 7:997-8. doi: 10.1111/irv.12064

15. Peng $\mathrm{Y}$, Xu Y, Zhu $\mathrm{M}, \mathrm{Yu} \mathrm{H}$, Nie $\mathrm{S}$, Yan $\mathrm{W}$. Chinese urban-rural disparity in pandemic (H1N1) 2009 vaccination coverage rate and associated determinants: a cross-sectional telephone survey. Public Health. (2013) 127:930-7. doi: 10.1016/j.puhe.2013.06.007

16. Lekana-Douki SE, Nkoghe D, Drosten C, Ngoungou EB, Drexler JF, Leroy EM. Viral etiology and seasonality of influenza-like illness in Gabon, March 2010 to June 2011. BMC Infect Dis. (2014) 14:373. doi: 10.1186/1471-2334-14-373

17. Kocik J, Niemcewicz M, Winnicka I, Michalski A, Bielawska-Drozd A, Kolodziej M, et al. Diversity of influenza-like illness etiology in Polish Armed Forces in influenza epidemic season. Acta Biochim Pol. (2014) 61:48994. doi: 10.18388/abp.2014_1869

18. Reed C, Chaves SS, Perez A, D'Mello T, Daily Kirley P, Aragon D, et al. Complications among adults hospitalized with influenza: a comparison of seasonal influenza and the $2009 \mathrm{H} 1 \mathrm{~N} 1$ pandemic. Clin Infect Dis. (2014) 59:166-74. doi: 10.1093/cid/ciu285

19. Dawood FS, Iuliano AD, Reed C, Meltzer MI, Shay DK, Cheng PY, et al. Estimated global mortality associated with the first 12 months of 2009 pandemic influenza A H1N1 virus circulation: a modelling study. Lancet Infect Dis. (2012) 12:687-95. doi: 10.1016/S1473-3099(12)70121-4

20. Mitchell R, Taylor G, McGeer A, Frenette C, Suh KN, Wong A, et al. Understanding the burden of influenza infection among adults in Canadian hospitals: a comparison of the 2009-2010 pandemic season with the prepandemic and postpandemic seasons. Am J Infect Control. (2013) 41:10327. doi: 10.1016/j.ajic.2013.06.008 
21. Yu H, Alonso WJ, Feng L, Tan Y, Shu Y, Yang W, et al. Characterization of regional influenza seasonality patterns in China and implications for vaccination strategies: spatio-temporal modeling of surveillance data. PLoS Med. (2013) 10:e1001552. doi: 10.1371/journal.pmed.10 01552

22. Saha S, Chadha M, Al Mamun A, Rahman M, Sturm-Ramirez K, Chittaganpitch $M$, et al. Influenza seasonality and vaccination timing in tropical and subtropical areas of southern and south-eastern Asia. Bull World Health Org. (2014) 92:318-30. doi: 10.2471/BLT.13.124412

23. Gao R, Cao B, Hu Y, Feng Z, Wang D, Hu W, et al. Human infection with a novel avian-origin influenza A (H7N9) virus. N Engl J Med. (2013) 368:1888-97. doi: 10.1056/NEJMoa1304459

24. Li Q, Zhou L, Zhou M, Chen Z, Li F, Wu H, et al. Epidemiology of human infections with avian influenza $\mathrm{A}(\mathrm{H} 7 \mathrm{~N} 9)$ virus in China. N Engl J Med. (2014) 370:520-32. doi: 10.1056/NEJMoa1304617

25. Terajima M, Babon JA, Co MD, Ennis FA. Cross-reactive human B cell and $\mathrm{T}$ cell epitopes between influenza A and B viruses. Virol J. (2013) 10:244. doi: 10.1186/1743-422X-10-244

26. Laurie KL, Carolan LA, Middleton D, Lowther S, Kelso A, Barr IG. Multiple infections with seasonal influenza $\mathrm{A}$ virus induce cross-protective immunity against $\mathrm{A}(\mathrm{H} 1 \mathrm{~N} 1)$ pandemic influenza virus in a ferret model. $J$ Infect Dis. (2010) 202:1011-20. doi: 10.1086/656188

27. Mugitani A, Ito K, Irie S, Eto T, Ishibashi M, Ohfuji S, et al. Immunogenicity of the trivalent inactivated influenza vaccine in young children less than 4 years of age, with a focus on age and baseline antibodies. Clin Vaccine Immunol. (2014) 21:1253-60. doi: 10.1128/CVI.00200-14
28. Sampath R, Russell KL, Massire C, Eshoo MW, Harpin V, Blyn LB, et al. Global surveillance of emerging Influenza virus genotypes by mass spectrometry. PLoS ONE. (2007) 2:e489. doi: 10.1371/journal.pone.0000489

29. Tramuto F, Maida CM, Magliozzo F, Amodio E, Vitale F. Occurrence of a case of influenza $\mathrm{A}(\mathrm{H} 1 \mathrm{~N} 1) \mathrm{pdm} 09$ and $\mathrm{B}$ co-infection during the epidemic season 2012-2013. Infect Genet Evol. (2014) 23:95-8. doi: 10.1016/j.meegid.2014.01.032

Conflict of Interest: The authors declare that the research was conducted in the absence of any commercial or financial relationships that could be construed as a potential conflict of interest.

Publisher's Note: All claims expressed in this article are solely those of the authors and do not necessarily represent those of their affiliated organizations, or those of the publisher, the editors and the reviewers. Any product that may be evaluated in this article, or claim that may be made by its manufacturer, is not guaranteed or endorsed by the publisher.

Copyright (๑ $2022 \mathrm{He}$, Hou, Chen, Yu, Chen, He, Liu, Gong, Huang, Wu, Pan and Gao. This is an open-access article distributed under the terms of the Creative Commons Attribution License (CC BY). The use, distribution or reproduction in other forums is permitted, provided the original author(s) and the copyright owner(s) are credited and that the original publication in this journal is cited, in accordance with accepted academic practice. No use, distribution or reproduction is permitted which does not comply with these terms. 